INLO-PUB-10/03; IFT-UAM/CSIC-03-29

\title{
Calorons and fermion zero-modes
}

\author{
Falk Bruckmann ${ }^{\mathrm{a}}$, Margarita García Pérez ${ }^{\mathrm{b}}$, Dániel Nógrádia and Pierre van Baal ${ }^{\mathrm{a}}$ * \\ anstituut-Lorentz for Theoretical Physics, University of Leiden,P.O. Box 9506, NL-2300 RA Leiden, \\ The Netherlands. \\ bInstituto de Física Teórica, C-XVI, Universidad Autónoma de Madrid, Madrid 28049, Spain.
}

Calorons in the confined phase for $\mathrm{SU}(n)$ gauge theory, having a non-trivial Polyakov loop, "dissolve" in $n$ monopole constituents for large enough instanton scale parameters. We discuss recent results for these caloron solutions and their fermion zero-modes, as well as the implications for lattice studies and comment on the possible influence of the constituent monopoles on the instanton size distribution.

\section{Introduction}

Calorons for $\mathrm{SU}(n)$, instantons at finite temperature, have $n$ monopoles as constituents 123 each with a mass $8 \pi^{2} \nu_{m} / \beta$, where $\nu_{m} \equiv \mu_{m+1}-\mu_{m}$, defined in terms of the Polyakov loop at spatial infinity (the so-called holonomy) through a global gauge rotation $g$ and its (ordered) eigenvalues,

$$
\begin{aligned}
& \mathcal{P}_{\infty}=g \exp \left[2 \pi i \operatorname{diag}\left(\mu_{1}, \ldots, \mu_{n}\right)\right] g^{\dagger}, \\
& \mu_{1} \leq \ldots \leq \mu_{n} \leq \mu_{n+1} \equiv 1+\mu_{1}, \quad \sum_{m=1}^{n} \mu_{m}=0 .
\end{aligned}
$$

Using the classical scale invariance we may set $\beta=1$ throughout. The charge one $\mathrm{SU}(n)$ action density has a simple form [3],

$$
\begin{aligned}
& \operatorname{Tr} F_{\mu \nu}^{2}(x)=\partial_{\mu}^{2} \partial_{\nu}^{2} \log \left[\frac{1}{2} \operatorname{tr}\left(\mathcal{A}_{n} \cdots \mathcal{A}_{1}\right)-\cos (2 \pi t)\right], \\
& \mathcal{A}_{m} \equiv \frac{1}{r_{m}}\left(\begin{array}{cc}
r_{m} & \left|\vec{y}_{m}-\vec{y}_{m+1}\right| \\
0 & r_{m+1}
\end{array}\right)\left(\begin{array}{cc}
c_{m} & s_{m} \\
s_{m} & c_{m}
\end{array}\right),
\end{aligned}
$$

with $r_{m}=\left|\vec{x}-\vec{y}_{m}\right|$ the center of mass radius of the $m^{\text {th }}$ constituent monopole, $c_{m} \equiv \cosh \left(2 \pi \nu_{m} r_{m}\right)$, $s_{m} \equiv \sinh \left(2 \pi \nu_{m} r_{m}\right), r_{n+1} \equiv r_{1}$ and $\vec{y}_{n+1} \equiv \vec{y}_{1}$.

The fermion zero-mode is localized 45] on the $m^{\text {th }}$ constituent monopole, if $\mu_{m}<z<\mu_{m+1}$ specifies its boundary condition $\Psi_{z}(t+\beta, \vec{x})=$ $\exp (-2 \pi i z) \mathcal{P}_{\infty} \Psi_{z}(t, \vec{x})$ in the so-called algebraic gauge, where $A_{\mu}(t+\beta, \vec{x})=\mathcal{P}_{\infty} A_{\mu}(t, \vec{x}) \mathcal{P}_{\infty}^{-1}$. "Cycling" through the values of $z$ gives a distinct signature, see e.g. Fig. 11 through which one can identify well-dissociated calorons, as observed for $\mathrm{SU}(2)$ [6] with, and for $\mathrm{SU}(3)$ [] without cooling.

\section{Progress at higher charge}

The analytic formalism can be generalized to higher charge calorons $(Q>1)$, although explicit results are harder to get at. In this section we report on work by three of us (FB, DN and PvB) 8 .

Periodically repeating $Q$ instantons (up to a colour rotation with $\mathcal{P}_{\infty}$ ) within each time-layer $t \in[p, p+1]$, using $p$ as a Fourier index, maps the appropriate ADHM data 9 to a self-dual $U(Q)$ gauge field $\hat{A}_{\mu}(z)$. This is defined on a circle parameterized by $z \in[0,1]$, with $n$ well-defined singularities at $z=\mu_{j}$, in accordance with the Nahm transformation [1]. When $\hat{A}(z)$ is constant for $z \in\left[\mu_{j}, \mu_{j+1}\right]$ (always true for $Q=1$ ), its eigenvalues are directly related to the constituent locations, all of the same type with a mass $8 \pi^{2} \nu_{j}$.

The motivation for these studies has been to understand to which degree the monopole constituents can be seen (outside their non-abelian cores) as independent point-like Dirac monopoles.

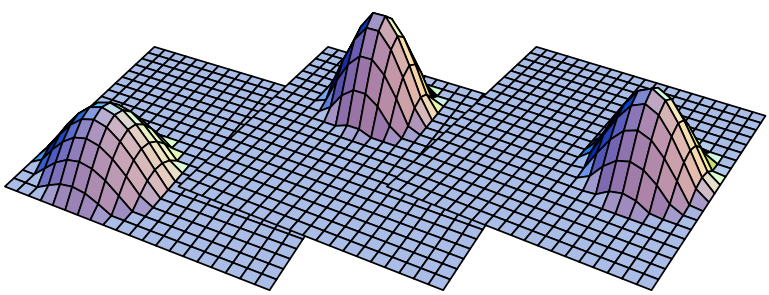

Figure 1. Logarithm of zero-mode densities with $z=4 / 5,1 / 2,1 / 5$ (from left to right) for an $\mathrm{SU}(3)$ caloron with $\left(\mu_{1}, \mu_{2}, \mu_{3}\right)=(-17,-2,19) / 60$. 
Approximate superpositions of calorons, e.g. using the sum ansatz [10, suffer from subtle effects that lead to finite energy remnants of the Dirac string as illustrated in Fig. 22 Fortunately this is an artefact of the approximation, as can be seen from a class of exact axially symmetric solutions, for which $\hat{A}(z)$ is piecewise constant. It does, however, make model building on the basis of these constituent monopoles intricate.

In the high temperature limit the non-abelian cores of the monopoles shrink to zero size. As long as $z \neq \mu_{j}$ the mass of the fermions becomes infinite in this limit and the zero-modes are entirely localized to the non-abelian cores of the constituent monopoles, cmp. Fig. 22 The fermion density, summed over all zero-modes, is given by $\sum_{a}\left|\Psi^{(a)}(x ; z)\right|^{2}=-(2 \pi)^{-2} \partial_{\mu}^{2} \operatorname{Tr} \hat{f}_{x}(z, z)$, where $\hat{f}_{x}\left(z, z^{\prime}\right)$ is the Green's function of the ADHMNahm construction and can be computed using impurity scattering methods. Many simplifications occur in the high temperature limit, where the density for $\mu_{m}<z<\mu_{m+1}$ can be written as $-\partial_{i}^{2} \mathcal{V}_{m}(\vec{x})$. This function can therefore be used to trace the possible extended nature of constituent monopole sources (independence of $z$ implies it is a highly non-trivial conserved quantity of the Nahm equations). For $\mathrm{SU}(2)$ and $Q=2$ we found

$\mathcal{V}_{m}(\vec{x})=\frac{1}{2 \pi|\vec{x}|}+\frac{\mathcal{D}}{4 \pi^{2}} \int_{r<\mathcal{D}} d r d \varphi \frac{\partial_{r}|\vec{x}-r \vec{y}(\varphi)|^{-1}}{\sqrt{\mathcal{D}^{2}-r^{2}}}$,

where $\vec{y}(\varphi)=\left(\sqrt{1-\mathbf{k}^{2}} \cos \varphi, 0, \sin \varphi\right)$, up to an arbitrary coordinate shift and rotation, in terms

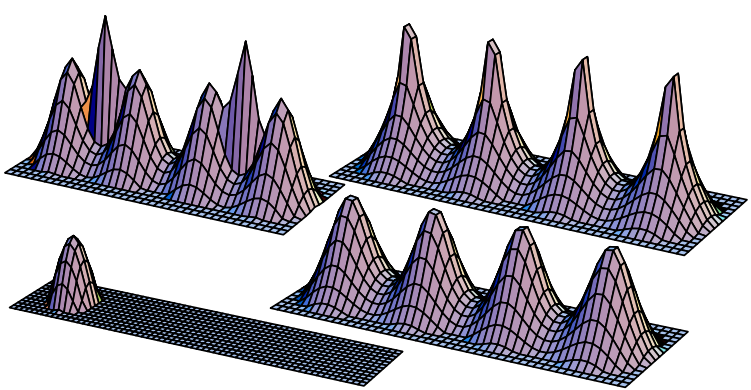

Figure 2. Logarithm of action densities for the sum ansatz (top-left), high temperature limit and exact (right) solutions of an $\mathrm{SU}(2)$ charge 2 caloron. Bottom-left corner shows one of the two anti-periodic $\left(z=\frac{1}{2}\right)$ zero-mode densities. of a scale $\mathcal{D}$ and shape parameter $\mathbf{k}$ to characterize arbitrary $\mathrm{SU}(2)$ charge 2 solutions of $\hat{A}(z)$.

This represents an extended structure with the core restricted to a disk bounded by an ellipse with minor axes $2 \mathcal{D} \sqrt{1-\mathbf{k}^{2}}$ and major axes $2 \mathcal{D}$. But for large $\mathcal{D}$ this can be shown to approach two point-like monopoles $(\mathbf{k} \rightarrow 1)$ separated by $2 \mathcal{D}$. Axially symmetric solutions are always point-like $(\mathbf{k}=1)$, with unlike-charges alternating.

\section{Truly observing constituents?}

We return to interpreting the recent lattice data of Ref. [7, based on using the fermion zeromodes as a filter, restricting to $\mathrm{Q}=1$ by insisting there is only one "exact" zero-mode. In the confined phase a reasonable fraction of configurations was found with one lump in the zero-mode density, but at different locations as a function of $z$. This fitted well the expected behaviour of a charge one SU(3) caloron, see Fig. 1 There will typically be near zero-modes as well, revealing the presence of $\mathrm{Q}$ anti- and $\mathrm{Q}+1$ instantons. To rule out that the zero-mode could be hopping between the $\mathrm{Q}+1$ instantons, rather than between the constituent monopoles of a single caloron, two of us (MGP and $\mathrm{PvB}$ ) computed the mixing of zeromodes as a function of $z$.

For simplicity we consider $\mathrm{SU}(2)$, adding a charge 1 anti-instanton and a charge 2 instanton in the algebraic gauge with (the same) arbitrary holonomy, i.e. using the sum ansatz 10. In isolation the charge 2 instanton has two exact zeromodes, and only one of them can survive: the one associated to the zero (left) eigenvector of the $2 \times 1$ overlap matrix [10] (using Weyl fermions)

$$
\begin{aligned}
O_{I A}^{i j}(z) & =\int d^{4} x \Psi_{(I)}^{(i)}(x ; z)^{\dagger} \sigma_{\mu} D_{\mu} \Psi_{(A)}^{(j)}(x ; z) \\
& =-\int d^{4} x \Psi_{(I)}^{(i)}(x ; z)^{\dagger} \sigma_{\mu} \partial_{\mu} \Psi_{(A)}^{(j)}(x ; z),
\end{aligned}
$$

where $\sigma_{\mu}=\left(\mathbb{1}_{2}, i \vec{\tau}\right)$ and the equation of motion for the zero-modes was used. We now assume that the charge 2 instanton is made up of two sufficiently separated instantons, in which case $\Psi_{(I)}^{(i)}$ to a good approximation is equal to the charge 1 zero-mode associated with the $i^{\text {th }}$ instanton.

The exact form of the $\mathrm{SU}(2) \mathrm{Q}=1$ zero-mode with $\mathcal{P}_{\infty}=\exp \left(2 \pi i \omega \tau_{3}\right)$ has been given before 4 . 
The anti-instanton and its zero-mode are obtained by time reversal, which changes the periodicity to $\mathcal{P}_{\infty}^{\dagger} \exp (2 \pi i z)$, to be corrected by a gauge rotation $i \tau_{2}$ and changing the sign of $z$. This ensures the overlap integral is periodic. In terms of the scale parameter $\rho$ (determining the distance $\pi \rho^{2} / \beta$ between the constituents), arbitrary location $a_{\mu}$ and orientation $R_{i j}=\frac{1}{2} \operatorname{tr}\left(U \tau_{i} U^{\dagger} \tau_{j}\right)$ (with $U \in \mathrm{SU}(2)$ the associated spin rotation), we find

$$
\begin{aligned}
& O_{I A}(z)=-\frac{\rho_{(I)} \rho_{(A)}}{4 \pi^{2}} \int d^{4} x\left(K(z)+K^{*}(-z)\right), \\
& K(z) \equiv \psi_{(I)}^{\dagger}\left(x^{(I)} ; z\right) U_{(I)}^{\dagger} \sigma_{\mu} \partial_{\mu} U_{(A)} \psi_{(A)}\left(x^{(A)} ; z\right),
\end{aligned}
$$

where, $x^{(I, A)}=\left(t-a_{0}^{(I, A)}, R^{(I, A)}\left(\vec{x}-\vec{a}^{(I, A)}\right)\right)$ and

$$
\begin{aligned}
& \psi_{(I)}(x ; z) \equiv \phi_{(I)}^{\frac{1}{2}}(x)\left(\begin{array}{c}
\partial_{2}+i \partial_{1} \\
\partial_{0}-i \partial_{3}
\end{array}\right) \hat{f}_{x}^{(I)}(\omega, z), \\
& \psi_{(A)}(x ; z) \equiv \phi_{(A)}^{\frac{1}{2}}(x)\left(\begin{array}{c}
\partial_{0}-i \partial_{3} \\
\partial_{2}-i \partial_{1}
\end{array}\right) \hat{f}_{x}^{(A)}(\omega, z) .
\end{aligned}
$$

Explicit expressions for $\phi(x)$ and $\hat{f}_{x}(\omega, z)$ can be found elsewhere 345].

For generic values of the parameters the overlap is complex and to go through zero (to maximize localization) requires fine-tuning of two parameters. We will assume here that, for each of the two instantons and the anti-instanton, both constituent monopoles are approximately at the same location $\left(\pi \rho^{2} / \beta\right.$ small $)$. Typically then, if one instanton is sufficiently further away from the anti-instanton their overlap will be exponentially small and the zero mode will localize on that instanton. This happens generically for any $z$ except for two small intervals (proportional to $\beta^{-1}$ ) around $z= \pm \omega$ where the (anti-)instanton zero mode becomes delocalized and the overlap is not guaranteed to be small. One could, in principle, observe in these two intervals a significant mixing with the zero mode of the second instanton. Notice however that, except for trivial holonomy where the two intervals merge into one (at $z=0$ or $\frac{1}{2}$ ), this effect leads to double the expected number of hoppings. Therefore it could only possibly mimic the behaviour of a single caloron with trivial holonomy. We can then conclude that the full signature of a single $\mathrm{SU}(2)$ caloron with nontrivial holonomy is unlikely to be emulated by the effect of hopping between two instantons, and we expect the same to hold for $\mathrm{SU}(3)$.

\section{Discussions}

Cooling studies at a low temperature, rather than just below the deconfinement temperature, have been reported as well [6]. Constituents at non-trivial holonomy could still be identified by points where two eigenvalues of the Polyakov loop coincide, but they revealed no individual lumps. This may explain why before no constituents were found. It is also interesting to point out that for $\rho>\beta$ the scale parameter plays an entirely different role and the measure for integration over the moduli space should be formulated in terms of constituent monopoles of fixed size (at fixed temperature). This should remove the infrared divergence of the scale integration, although it is unlikely a semiclassical picture can be used here.

\section{Acknowledgements}

We thank Michael Müller-Preussker, Michael Ilgenfritz and Christof Gattringer for discussions. FB is supported by FOM and MGP by MCYT.

\section{REFERENCES}

1. W. Nahm, Self-dual monopoles and calorons, in: Lecture Notes in Physics 201 (1984) 189.

2. K.Lee and P. Yi, Phys. Rev. D56(1997)3711; K.Lee and C.Lu, Phys.Rev.D58(1998)025011.

3. T.C. Kraan and P. van Baal, Nucl. Phys. B533 (1998) 627; Phys. Lett. B435 (1998) 389.

4. M.García Pérez, A.González-Arroyo, C.Pena and P. van Baal, Phys.Rev. D60(1999)031901.

5. M.N. Chernodub, T.C. Kraan, P. van Baal, Nucl. Phys. B(Proc.Suppl.)83-84(2000)556.

6. E.-M. Ilgenfritz et al., Phys. Rev. D66 (2002) 074503; E.-M. Ilgenfritz, talk presented at this conference.

7. C. Gattringer, Phys. Rev. D67 (2003) 034507; C. Gattringer and S. Schaefer, Nucl. Phys. B654 (2003) 30.

8. F. Bruckmann, P. van Baal, Nucl. Phys. B653 (2002) 105; F. Bruckmann, D. Nógrádi, P. van Baal, Nucl. Phys. B666 (2003) 195.

9. M.F. Atiyah, N.J. Hitchin, V. Drinfeld and Yu.I. Manin, Phys. Lett. 65A (1978) 185.

10. T. Schäfer and E.V. Shuryak, Rev.Mod.Phys. 70 (1998) 323; D. Diakonov, hep-ph/0212026 